\title{
Hidden Wall Supersymmetry Breakdown
}

\author{
Hans Peter Nilles \\ Physikalisches Institut, Universität Bonn, Nussallee 12, D-53115 Bonn, Germany \\ E-mail: 'nilles@dirac.physik.uni-bonn.de!
}

Abstract: The M-theoretic extension of the heterotic $E_{8} \times E_{8}$ string leads to a geometrical picture of two walls (branes) at the ends of a finite 11-dimensional interval. While the supergravity multiplet can penetrate in the $d=11$ bulk, the two $E_{8}$ gauge multiplets are confined to the two walls, respectively. We consider hidden sector supersymmetry breakdown in the framework of this theory. Gravitational interactions are shown to induce soft breaking terms in the observable sector; generically all of order of the gravitino mass. This is very similar to the situation in the weakly coupled case, with a difference for the observable sector gaugino masses that leads to important phenomenological and cosmological consequences.

\section{Introduction}

In the past, a discussion of the phenomenological applications of string theory has mainly concentrated on the weakly coupled heterotic $E_{8} \times E_{8}$ string. Models with realistic gauge groups and particle content have been constructed. Qualitatively the unification of gauge and gravitational couplings can be understood [i-1] with some moderate uncertainties in the relation between the string scale $M_{\text {string }}$ and the grand unified scale $M_{\mathrm{GUT}}$. Supersymmetry is broken dynamically via gaugino condensation den sector supergravity model at low energies with specific predictions for the soft breaking parameters including problematically small gaugino masses [i $[\bar{i}]$. At the moment we do not possess a convincing mechanism to resolve this problem.

Recently there have been attempts to study string theories in the region of intermediate and strong coupling. The strongly coupled version of the $E_{8} \times E_{8}$ theory is believed to be an orbifold of 11-dimensional M-theory, an interval in $d=11$ with $E_{8} \times E_{8}$ gauge fields restricted to the two $d=10$ dimensional boundaries respectively [5]. Applied to the question of unification $\left[6_{1}^{6}\right]$ the following picture emerges: the GUT- scale $M_{\mathrm{GUT}}=3 \times 10^{16} \mathrm{GeV}$ is identified with $1 / R$ where $V=R^{6}$ is the volume of compactified six-dimensional space. $\alpha_{\mathrm{GUT}}=1 / 25$ and the correct value of the $d=4$ reduced Planck mass $M_{P}=2.4 \times 10^{18} \mathrm{GeV}$ can be obtained by choosing the length of the $d=11$ interval to be $R_{11} \approx 6 R$. The fundamental mass scale of the $d=11$ theory $M_{11}=\kappa^{-2 / 9}$ (with $\kappa$ the $d=11$ Einstein gravitational coupling) has to be chosen roughly a factor 2 larger than $M_{\mathrm{GUT}}$ and at that scale $\alpha_{\text {string }}=g_{\text {string }}^{2} / 4 \pi$ is of order unity. This then represents a rather natural framework for the unification of coupling constants. The large compactification radius could lead to a solution

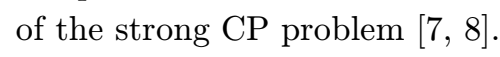

As in the weakly coupled theory, supersymmetry might be broken dynamically by gaugino condensation in the hidden $E_{8}$ on one boundary

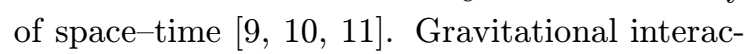
tions will play the role of messengers to the observable sector at the opposite boundary with interesting consequences for the soft supersymmetry breaking parameters $\left[10_{1}^{1}, i_{1}^{1}\right.$. In this talk we shall discuss this mechanism in detail and compute the predictions for the low energy effective theory. We find results very similar to the situation in the $d=10$ weakly coupled case, with one notable exception: gaugino masses are of comparable size to the gravitino mass, thus solving the 
problem of small gaugino masses that occurred in the weakly coupled case. The smallness of the gaugino masses in the observable sector has always been problematic, both for explicit model building and also in connection with the desire to obtain a realistic cold dark matter candidate in form of a stable neutralino. The strongly coupled version avoids these problems in a natural way.

\section{Gaugino condensation}

In our review of gaugino condensation in the $d=$ 10 weakly coupled $E_{8} \times E_{8}$ theory we shall start from the $d=10$ effective field theory and go to $d=4$ dimensions via the method of reduction and truncation explained in ref. [1 121 . In string theory compactified on an orbifold this would describe the dynamics of the untwisted sector. We retain the usual moduli fields $S$ and $T$ as well as matter fields $C_{i}$ that transform nontrivially under the observable sector gauge group. In this approximation, the Kähler potential is given by

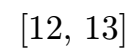

$G=-\log (S+\bar{S})-3 \log \left(T+\bar{T}-2 C_{i} \bar{C}_{i}\right)+\log |W|^{2}$

with superpotential

$$
W(C)=d_{i j k} C_{i} C_{j} C_{k}
$$

and the gauge kinetic function is given by the dilaton field

$$
f=S .
$$

We assume the formation of a gaugino condensate $\langle\chi \chi\rangle=\Lambda^{3}$ in the hidden sector where $\Lambda$ is the renormalization group invariant scale of the confining hidden sector gauge group. The gaugino condensate appears in the expression for the auxiliary components of the chiral superfields [1]

$$
F_{j}=\left(G^{-1}\right)_{j}^{k}\left(\exp (G / 2) G_{k}+\frac{1}{4} f_{k}(\chi \chi)\right)+\ldots
$$

which are order parameters for supersymmetry breakdown. Minimizing the scalar potential we find $F_{S}=0, F_{T} \neq 0$ and a vanishing cosmological constant. Supersymmetry is broken and the gravitino mass is given by [1]

$$
m_{3 / 2}=\frac{\left\langle F_{T}\right\rangle}{T+\bar{T}} \approx \frac{\Lambda^{3}}{M_{P}^{2}}
$$

and $\Lambda=10^{13} \mathrm{GeV}$ would lead to a gravitino mass in the $\mathrm{TeV}$ - range. A first inspection of the soft breaking terms in the observable sector gives a disturbing result. They vanish in this approximation. Scalar masses are zero because of the noscale structure in (2.1) (coming from the fact that we have only included fields of modular weight

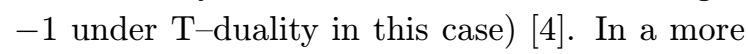
general situation we would get scalar masses $m_{0}$ comparable to the gravitino mass $m_{3 / 2}$ and the above result $m_{0}=0$ is just an artifact of the chosen approximation at the classical level. Gaugino masses $m_{1 / 2}$ are given by

$$
m_{1 / 2}=\frac{\frac{\partial f}{\partial S} F_{S}+\frac{\partial f}{\partial T} F_{T}}{2 \operatorname{Re} f}
$$

and with $f=S$ and $F_{S}=0$ we obtain $m_{1 / 2}=$ 0 . One loop corrections will change this picture as can be seen already by an inspection of the Green-Schwarz anomaly cancellation counter terms, as they modify $f$ at one loop. In the simple example of the so-called standard embedding with gauge group $E_{6} \times E_{8}$ we obtain [1는,

$$
f_{6}=S+\epsilon T ; \quad f_{8}=S-\epsilon T .
$$

This dependence of $f$ on $T$ will via (2.6i) lead to nonvanishing gaugino masses which, however, will be small compared to $m_{3 / 2}$ and $m_{0}$ since $\epsilon T$ is considered a small correction to the classical result. This might be problematic when applied to the supersymmetric extension of the standard model. With the large difference of the soft scalar and the gaugino masses a sizeable fine tuning is needed to induce the breakdown of electroweak symmetry at the correct scale [i1 $\overline{7}]$. The smallness of the gaugino masses also leads to an apparent problem in the context of relic abundances of the

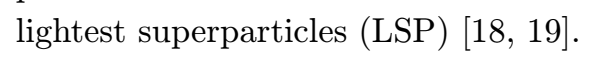

\section{Heterotic M-theory}

Let us now reconsider these questions in the strongly coupled $E_{8} \times E_{8}-M$-theory, which we shall discuss now in detail. The effective action is given 
by (for details see [iñ

$$
\begin{gathered}
L=\frac{1}{\kappa^{2}} \int d^{11} x \sqrt{g}\left[-\frac{1}{2} R+\ldots\right]+\ldots \\
+\frac{1}{2 \pi\left(4 \pi \kappa^{2}\right)^{2 / 3}} \int d^{10} x \sqrt{g}\left[-\frac{1}{4} F_{A B}^{a} F^{a A B}+\ldots\right] .
\end{gathered}
$$

Compactifying to $d=4$ we obtain [i6] (with the

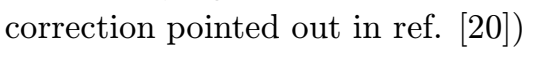

$$
\begin{aligned}
G_{N} & =8 \pi \kappa_{4}^{2}=\frac{\kappa^{2}}{8 \pi^{2} V \rho}, \\
\alpha_{G U T} & =\frac{\left(4 \pi \kappa^{2}\right)^{2 / 3}}{V}
\end{aligned}
$$

with $V=R^{6}$ and $\pi \rho=R_{11}$. Fitting $G_{N}$ and $\alpha_{G U T}=1 / 25$ then gives $R_{11} M_{11}$ of order 10 and $M_{11} R \approx 2.3$. The rather large value of the $d=4$ reduced Planck Mass $M_{P}=\kappa_{4}^{-1}$ is obtained as a result of the fact that $R_{11}$ is large compared to $R$.

We now perform a compactification using the method of reduction and truncation as above. For the metric we write [1뭉,

$$
g_{M N}=\left(\begin{array}{lll}
e^{-\gamma} e^{-2 \sigma} g_{\mu \nu} & & \\
& e^{\sigma} \delta_{m n} & \\
& & e^{2 \gamma} e^{-2 \sigma}
\end{array}\right)
$$

with $M, N=1 \ldots 11 ; \mu, \nu=1 \ldots 4 ; m, n=$ $5 \ldots 10 ; 2 R_{11}=2 \pi \rho=M_{11}^{-1} e^{\gamma} e^{-\sigma}$ and $V=$ $e^{3 \sigma} M_{11}^{6}$. At the classical level this leads to a Kähler potential as in (2.1i)

$$
K=-\log (\mathcal{S}+\overline{\mathcal{S}})-3 \log \left(\mathcal{T}+\overline{\mathcal{T}}-2 C_{i} \bar{C}_{i}\right)
$$

with

$$
\begin{aligned}
& \mathcal{S}=\frac{2}{(4 \pi)^{2 / 3}}\left(e^{3 \sigma} \pm i 24 \sqrt{2} D\right), \\
& \mathcal{T}=\frac{\pi^{2}}{(4 \pi)^{4 / 3}}\left(e^{\gamma} \pm i 6 \sqrt{2} C_{11}\right)
\end{aligned}
$$

where $D$ and $C_{11}$ fields are defined by

$$
\begin{aligned}
\frac{1}{4 !} e^{6 \sigma} G_{11 \lambda \mu \nu} & =\epsilon_{\lambda \mu \nu \rho}\left(\partial^{\rho} D\right), \\
C_{11 i \bar{j}} & =C_{11} \delta_{i \bar{j}}
\end{aligned}
$$

and $x^{i}\left(x^{\bar{j}}\right)$ is the holomorphic (antiholomorphic) coordinate of the Calabi-Yau manifold. The imaginary part of $\mathcal{S}(\operatorname{Im} \mathcal{S})$ corresponds to the model independent axion, and the gauge kinetic function is $f=\mathcal{S}$. This is very similar to the weakly coupled case. Before drawing any conclusion from these formulae, however, we have to discuss a possible obstruction at the one loop level. It can be understood from the mechanism of anomaly cancellation [i] in $d=10$ supergravity to be well defined one has to satisfy $d H=\operatorname{tr} F_{1}^{2}+\operatorname{tr} F_{2}^{2}-\operatorname{tr} R^{2}=0$ cohomologically. In the simplest case of the standard embedding one assumes $\operatorname{tr} F_{1}^{2}=\operatorname{tr} R^{2}$ locally and the gauge group is broken to $E_{6} \times E_{8}$. Since in the M-theory case the two different gauge groups live on the two different boundaries of space-time such a cancellation point by point is no longer possible. We expect nontrivial vacuum expectation values (vevs) of

$$
(d G) \propto \sum_{i} \delta\left(x^{11}-x_{i}^{11}\right)\left(\operatorname{tr} F_{i}^{2}-\frac{1}{2} \operatorname{tr} R^{2}\right)
$$

at least on one boundary ( $x_{i}^{11}$ is the position of $i_{-}$ th boundary). In the case of the standard embedding we would have $\operatorname{tr} F_{1}^{2}-\frac{1}{2} \operatorname{tr} R^{2}=\frac{1}{2} \operatorname{tr} R^{2}$ on one and $\operatorname{tr} F_{2}^{2}-\frac{1}{2} \operatorname{tr} R^{2}=-\frac{1}{2} \operatorname{tr} R^{2}$ on the other boundary. This might pose a severe problem since a nontrivial vev of $G$ might be in conflict with supersymmetry $\left(G_{11 A B C}=H_{A B C}\right)$. The supersymmetry transformation law in $d=11$ reads

$\delta \psi_{M}=D_{M} \eta+\frac{\sqrt{2}}{288} G_{I J K L}\left(\Gamma_{M}^{I J K L}+\ldots\right) \eta+\ldots$

Supersymmetry will be broken unless e.g. the derivative term $D_{M} \eta$ compensates the nontrivial vev of $G$. Witten has shown [i] that such a cancellation can occur and constructed the solution in the linearized approximation (linear in the expansion parameter $\kappa^{2 / 3}$ ) which corresponds to the large $T$-limit in the weakly coupled theory*. The supersymmetric solution can be shown to lead [100"] to a nontrivial dependence of the $\sigma$ and $\gamma$ fields with respect to $x^{11}$ :

$$
\frac{\partial \gamma}{\partial x^{11}}=-\frac{\partial \sigma}{\partial x^{11}}=\frac{\sqrt{2}}{24} \frac{\int d^{6} x \sqrt{g} \omega^{A B} \omega^{C D} G_{A B C D}}{\int d^{6} x \sqrt{g}}
$$

where the integrals are over the Calabi-Yau manifold and $\omega$ is the corresponding Kähler form. Formula (3.1. $\overline{1}$ - $)$ contains all the information to

\footnotetext{
*For a discussion beyond this approximation in the weakly coupled case see ref. [21].
} 
deduce the effective $d=4$ supergravity theory, including the Kähler potential and the gauge ki-

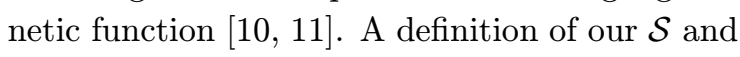
$\mathcal{T}$ fields in the four-dimensional theory now requires an average over the 11-dimensional interval. We therefore write

$$
\begin{aligned}
\mathcal{S} & =\frac{2}{(4 \pi)^{2 / 3}}\left(e^{3 \bar{\sigma}} \pm i 24 \sqrt{2} \bar{D}\right), \\
\mathcal{T} & =\frac{\pi^{2}}{(4 \pi)^{4 / 3}}\left(e^{\bar{\gamma}} \pm i 6 \sqrt{2} \bar{C}_{11}\right)
\end{aligned}
$$

where bars denote averaging over the 11th dimension. It might be of some interest to note that the combination $\mathcal{S} \mathcal{T}^{3}$ is independent of $x^{11}$ even before this averaging procedure took place.

$\exp (3 \sigma)$ represents the volume of the sixdimensional compact space in units of $M_{11}^{-6}$. The $x^{11}$ dependence of $\sigma$ then leads to the geometrical picture that the volume of this space varies with $x^{11}$ and differs at the two boundaries. In the given approximation, this variation is linear, and for growing $R_{11}$ the volume on the $E_{8}$ side becomes smaller and smaller. At a critical value of $R_{11}$ the volume will thus vanish and this will provide us with an upper limit on $R_{11}$. For the phenomenological applications we then have to check whether our preferred choice of $R_{11}$ that fits the correct value of the $d=4$ Planck mass ${ }^{\dagger}$ satisfies this bound. Although the coefficients are model dependent we find in general that the bound can be satisfied, but that $R_{11}$ is quite close to its critical value. A choice of $R_{11}$ much larger than $\left(\text { few } \times 10^{15} \mathrm{GeV}\right)^{-1}$ is therefore not permitted.

This variation of the volume is the analogue of the one loop correction of the gauge kinetic function (2.7) in the weakly coupled case and has the same origin, namely a Green-Schwarz anomaly cancellation counterterm. In fact, also in the strongly coupled case we find [i10 consequence of $\left(\overline{3}_{1}\right)$, corrections for the gauge coupling constants at the $E_{6}$ and $E_{8}$ side.

Gauge couplings will no longer be given by the (averaged) $\mathcal{S}$-field, but by that combination

\footnotetext{
${ }^{\dagger}$ With $V$ depending on $x^{11}$ we have to specify which values should be used in eq. $(\overline{3} \cdot \overline{3})$. The appropriate choice in the expression for $G_{N}$ is the average value of $V$ while in the expression for $\alpha_{G U T}$ we have to use $V$ evaluated at the $E_{6}$ border.
}

of the (averaged) $\mathcal{S}$ and $\mathcal{T}$ fields which corresponds to the $\mathcal{S}$-field before averaging at the given boundary:

$$
f_{6,8}=\mathcal{S} \pm \alpha \mathcal{T}
$$

at the $E_{6}\left(E_{8}\right)$ side respectively ${ }^{\ddagger}$. The critical value of $R_{11}$ will correspond to infinitely strong coupling at the $E_{8}$ side $\mathcal{S}-\alpha \mathcal{T}=0$ (Notice the similarity to (2.7) in the weakly coupled limit). Since we are here close to criticality a correct phenomenological fit of $\alpha_{\mathrm{GUT}}=1 / 25$ should include this correction $\alpha_{\mathrm{GUT}}^{-1}=\mathcal{S}+\alpha \mathcal{T}$ where $\mathcal{S}$ and $\alpha \mathcal{T}$ give comparable contributions. This is a difference to the weakly coupled case, where in $f=S+\epsilon T$ the latter contribution was small compared to $S$. Observe that this picture of a loop correction $\alpha \mathcal{T}$ to be comparable to the tree level result still makes sense in the perturbative expansion, since $f$ does not receive further per-

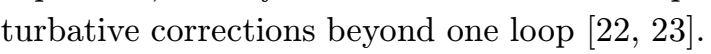

\section{Supersymmetry breakdown in $\mathrm{M}-$ theory}

In a next step we are now ready to discuss the dynamical breakdown of supersymmetry via gaugino condensation in the strongly coupled M-theory picture. In analogy to the previous discussion we start investigating supersymmetry transformation laws in the higher-dimensional (now $d=$ 11) field theory [igin]:

$$
\begin{aligned}
\delta \psi_{A} & \sim D_{A} \eta+G_{I J K L}\left(\Gamma_{A}^{I J K L}-8 \delta_{A}^{I} \Gamma^{J K L}\right) \eta \\
& -\delta\left(x^{11}\right)\left(\bar{\chi}^{a} \Gamma_{B C D} \chi^{a}\right) \Gamma_{A}^{B C D} \eta+\ldots(4.1) \\
\delta \psi_{11} & \sim D_{11} \eta+G_{I J K L}\left(\Gamma_{11}^{I J K L}-8 \delta_{11}^{I} \Gamma^{J K L}\right) \eta \\
& +\delta\left(x^{11}\right)\left(\bar{\chi}^{a} \Gamma_{A B C} \chi^{a}\right) \Gamma^{A B C} \eta+\ldots
\end{aligned}
$$

where gaugino bilinears appear in the right hand side of both expressions. It can therefore be expected that gaugino condensation breaks supersymmetry. Still the details have to be worked out. In the $d=10$ example, the gaugino condensate and the three-index tensor field $H$ contributed to the scalar potential in a full square. This lead to a vanishing cosmological constant as well as the fact that $F_{S}=0$ at the classical level.

\footnotetext{
$\ddagger$ With the normalization of the $\mathcal{T}$ field as in (3.14), $\alpha$ is a quantity of order 1 .
} 
Hořava has observed [י̄in that a similar mechanism might be in operation in the $d=11$ theory After a careful calculation this leads to a vanishing variation $\delta \psi_{A}=0$. In our model (based on reduction and truncation) we can now compute these quantities explicitly. We assume gaugino condensation to occur at the $E_{8}$ boundary

$$
\left\langle\bar{\chi}^{a} \Gamma_{i j k} \chi^{a}\right\rangle=\Lambda^{3} \epsilon_{i j k}
$$

where $\Lambda<M_{\mathrm{GUT}}$ and $\epsilon_{i j k}$ is the covariantly constant holomorphic 3-form. This leads to a nontrivial vev of $G_{11 A B C}$ at this boundary and supersymmetry is broken ${ }^{\S}$. At that boundary we obtain $F_{\mathcal{S}}=0$ and $F_{\mathcal{T}} \neq 0$ as expected from the fact that the component $\psi_{11}$ of the $11-$ dimensional gravitino plays the role of the goldstino.

In the effective $d=4$ theory we now have to average over the 11th dimension leading to

$$
\left\langle F_{\mathcal{T}}\right\rangle \approx \frac{1}{2} \mathcal{T} \frac{\int d x^{11} \delta \psi_{11}}{\int d x^{11}}
$$

as the source of SUSY breakdown.

This will then allow us to compute the size of supersymmetry breakdown on the observable $E_{6}$ side. Gravitational interactions play the role of messengers that communicate between the two boundaries. This effect can be seen from (3.3): large $R_{11}$ corresponds to large $M_{P}$ and $\left\langle F_{\mathcal{T}}\right\rangle$ gives the effective size of SUSY breaking on the $E_{6}$ side $\left(R_{11} \rightarrow \infty\right.$ implies $\left.M_{P} \rightarrow \infty\right)$. The gravitino mass is given by

$$
m_{3 / 2}=\frac{\left\langle F_{\mathcal{T}}\right\rangle}{\mathcal{T}+\overline{\mathcal{T}}} \approx \frac{\Lambda^{3}}{M_{P}^{2}}
$$

(similar to $(\underline{2} . \overline{5})$ in the weakly coupled case) and we expect this to represent the scale of soft supersymmetry breaking parameters in the observable sector [1] 그. These soft masses are determined by the coupling of the corresponding fields to the goldstino multiplet. As we have seen before, we cannot compute the scalar masses reliably in our approximation: $m_{0}=0$ because of the no-scale

\footnotetext{
§One might speculate that a nontrivial vev of $D_{A} \eta$ might be operative here as in the case without gaugino condensation (see discussion after eq. (B.11 1 )). However, the special values of $H_{i j k} \propto \epsilon_{i j k}$ necessary to cancel the contribution of the gaugino condensate do not permit such a mechanism (see footnote 6 in ref. ['6i]).
}

structure that appears as an artifact of our approximation. Fields of different modular weight will receive contribution to $m_{0}$ of order $m_{3 / 2}$. For the mass of a field $C$ we have [1]

$$
m_{0}^{2}=m_{3 / 2}^{2}-F^{i} \bar{F}^{\bar{j}} \frac{Z_{i \bar{j}}-Z_{i} Z^{-1} \bar{Z}_{\bar{j}}}{Z}
$$

where $i, j=\mathcal{S}, \mathcal{T}$ and $Z$ is the moduli dependent coefficient of $C \bar{C}$ term appearing in the Kähler potential. Scalars of modular weight -1 will become massive through radiative corrections. This then leads to the expectation that $m_{3 / 2}$ should be in the $\mathrm{TeV}$-region and $\Lambda \approx 10^{13} \mathrm{GeV}$ ?. So far this is all similar to the weakly coupled case.

An important difference appears, however, when we turn to the discussion of observable sector gaugino masses $(2.6 i)$. In the weakly coupled case they were zero at tree level and appeared only because of the radiative corrections at one loop (2.7). As a result of this small correction, gaugino masses were expected to be much smaller than $m_{3 / 2}$. In the strongly coupled case the ana$\log$ of $(2.6)$ is still valid

$$
m_{1 / 2}=\frac{\frac{\partial f_{6}}{\partial \mathcal{S}} F_{\mathcal{S}}+\frac{\partial f_{6}}{\partial \mathcal{T}} F_{\mathcal{T}}}{2 \operatorname{Re} f_{6}}
$$

and the 1-loop effect is encoded in the variation of the $\sigma$ and $\gamma$ fields from one boundary to the other. Here, however, the loop corrections are sizable compared to the classical result because of the fact that $R_{11}$ is close to its critical value. As a result we expect observable gaugino masses of the order of the gravitino mass. The problem of the small gaugino masses does therefore not occur in this situation. Independent of the question whether $F_{\mathcal{S}}$ or $F_{\mathcal{T}}$ are the dominant sources of supersymmetry breakdown, the gauginos will be heavy of the order of the gravitino mass. The exact relation between the soft breaking parameters $m_{0}$ and $m_{1 / 2}$ will be a question of model building. If in some models $m_{0} \ll m_{1 / 2}$ this might give a solution to the flavor problem. The no-scale structure found above might be a reason for such a suppression of $m_{0}$. As we have discussed above, this structure, however, is an artifact of our simplified approximation and does

IIn realistic models $E_{8}$ is broken and $\Lambda$ is adjusted by model building. 
not survive in perturbation theory. At best it could be kept exact (but only for the fields with modular weight -1 ) in the $R_{11} \rightarrow \infty$ limit. The upper bound on $R_{11}$ precludes such a situation. With observable gaugino masses of order $m_{3 / 2}$ we also see that $m_{3 / 2}$ cannot be arbitrarily large

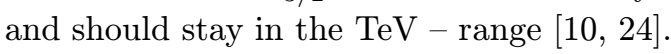

\section{The Super-Higgs mechanism}

In the previous sections the estimate of the gravitino mass was obtained $[11 \overline{1} 1,0$ simplified approximation according to which the higher dimensional bulk fields were integrated out via an averaging proceedurell. In this picture, the goldstino mode was represented by the lowest Kaluza-Klein $\Psi_{0}$ mode of a higher dimensional field $\Psi$. In the super-Higgs mechanism this mode supplies the additional degrees of freedom to render the gravitino massive. Qualitatively this simplified approximation does give a consistent picture, but there remain some open questions and potential problems when one looks into details of the super-Higgs mechanism. In this section we would like to point out these potential problems and show how they can be resolved. For a detailed explanation we refer the reader to [2] $2 \overline{8}]$. We shall concentrate here on a qualitative discussion of the mechanism involved. This will include a discussion of the possible nature of the goldstino (is it a bulk or a wall field), the relation to the Scherk-Schwarz mechanism [209"] in that context [30i] and an upper limit for the gravitino mass in the present picture. We shall argue that a meaningful realization of the super-Higgs mechanism seems to require some modes in the bulk other than the graviton and the gravitino. Finally we shall comment on the phenomenological consequences of this findings, including a discussion of the nature of the soft breaking terms on both walls.

Specifically we want to address the following two questions:

(i) the nature of the massless gravitino in the

\footnotetext{
I A corresponding analysis in global supersymmetry has been performed in ref. [26i]. Related work in the supergravity case has been given in [27].
}

presence of several $F$-terms** on different walls that cancel and lead to unbroken supersymmetry

(ii) the identification of the goldstino in the case of broken supersymmetry.

The first question (i) arises because of a particalur nonlocal effect of supersymmetry breakdown first observed by Horava. A given source of supersymmetry breakdown (parametrized by a vacuum expectation value (vev) of an auxiliary field $F$ ) on one wall could be compensated by a similar but opposite value $(-F)$ on another (separated) wall. Any calculation and approximation of the system thus has to reproduce this behaviour. The previously mentioned averaging proceedure over the bulk distance does this in a trivial way, leading to unbroken supersymmetry as expected. A detailed inspection of the gravitino, however, reveals a problem. If we start with the situation $F=0$ it is easy to define the massless gravitino $\Psi_{0}$ in the $d=4$ theory. Switching on a nontrivial $F$ on one brane and $(-F)$ on the other still should give a massless gravitino, but $\Psi_{0}$ turns out to be no longer a mass eigenstate. The resolution of this problem and the correct identification of the gravitino can be found in [28] of the possible gravitini that appear when one, for example, reduces a 5-dimensional theory to a theory in $d=4$ on a finite $d=5$ interval. The theory on a $d=5$ circle would lead to $N=2$ supersymmetry in $d=4$ and two massless gravitini (zero modes on the circle). The $Z_{2}$ projection on the interval removes one of the gravitini and is $N=1$ supersymmetric. A nonvanishing vev of $F$ now interferes with the boundary conditions and the massless gravitino will be a linear combination of the zero mode and all the excited KaluzaKlein modes whose coefficients will depend on $F$ (assuming, of course, unbroken supersymmetry due to a compensating vev $-F$ on another wall).

The second question (ii) deals with the nature of the goldstino (i.e. the longitudinal components of the gravitino) in the case of broken

\footnotetext{
**We generically use the notation $F$-term for the source of supersymmetry breakdown. Depending on the specific situation this could represent a $D$-term or a gaugino condensate as well.
} 
supersymmetry. Remember that the simplified averaging proceedure leads to a goldstino that corresponds to the lowest Kaluza-Klein mode $\Psi_{0}$ of a higher-dimensional bulk field $\Psi$. Inspecting the gravitino mass matrix in this case reveals the fact that this field $\Psi_{0}$ is not a mass eigenstate, but mixes with infinitely many higher KaluzaKlein modes $\Psi_{n}$. A consistent manifestation of a super-Higgs mechanism would require a diagonalization of this mass matrix and an identification of the goldstino. This problem has been solved in [28]'], by a suitable redefinition of the Kaluza-Klein modes. Thus it is shown that a consistent Super-Higgs mechanism is at work.

\section{Conclusions}

This resolution of these puzzles clarifies some of the other questions of the approach.

- The nonlocality of the breakdown shows some resemblance to the breakdown of supersymmetry via the Scherk-Schwarz [2 $2 \overline{2}]$ mechanism. Here, however, the real goldstino of the spontaneous breakdown of supersymmetry can be unambiguously identified.

- The possibility to cancel the supersymmetry breakdown on a distant wall by a vev on the local wall tells us, that the mass splittings of broken supersymmetry have to be of order of the gravitino mass $m_{3 / 2}$ on both walls.

- In terms of the physical quantities there is no real extra suppression, once we separate the walls by a large distance $R$. In the limit $R \rightarrow \infty$ we will have $M_{\text {Planck }} \rightarrow \infty$ as well. The suppression of the soft breaking parameters will always be gravitational.

- In general, when we have a system of many separated branes with potential sources of supersymmetry breakdown, the actual breakdown will be obtained by the sum of these contributions. The averaging proceedure will be very useful to decide whether supersymmetry is broken or not. The identification of the goldstino, however, is more difficult and requires a careful calculation.
- A successful implementation of the superHiggs mechanism will require some fields other than gravitino and graviton in the bulk $^{\dagger \dagger}$. This implies that in the absence of such fields a consistent spontaneous breakdown of supergravity might not be achieved.

To summarize we can say that the picture of supersymmetry breakdown in the M-theoretic limit looks very promising. It is very similar to the weakly coupled case, but avoids the problem of the small gaugino masses. This has important consequences for the phenomenological and cosmological properties of the effective models in four dimensions.

\section{Acknowledgements}

I would like to thank Y. Kawamura, K. Meissner, M. Olechowski and M. Yamaguchi for useful discussions and collaboration. This work has been supported by the European Commission program ERBFMRX-CT96-0090.

\section{References}

[1] D.J. Gross, J.A. Harvey, E. Martinec and R. Rohm, Nucl. Phys. B267 (1986) 75.

[2] J.P. Derendinger, L.E. Ibáñez and H.P. Nilles, Phys. Lett. B155 (1985) 65.

[3] M. Dine, R. Rohm, N. Seiberg and E. Witten, Phys. Lett. B156 (1985) 55.

[4] A. Brignole, L.E. Ibáñez and C. Muñoz, Nucl. Phys. B422 (1994) 125.

[5] P. Hořava and E. Witten, Nucl. Phys. B460 (1996) 506; Nucl. Phys. B475 (1996) 94.

[6] E. Witten, Nucl. Phys. B471 (1996) 135.

[7] T. Banks and M. Dine, Nucl. Phys. B479 (1996) 173.

[8] K. Choi, hep-th/9706171, Phys. Rev. D56 (1997) 6588

[9] P. Hořava, Phys. Rev. D54 (1996) 7561.

[10] H. P. Nilles, M. Olechochowski and M. Yamaguchi, hep-th/9707143, Phys. Lett. B415 (1997) 24

\footnotetext{
$\dagger^{\dagger}$ Usually they arise as modes of the higher dimensional supergravity multiplet.
} 
[11] H. P. Nilles, M. Olechochowski and M. Yamaguchi, hep-th/9801030, Nucl. Phys. B530 (1998) 43

[12] E. Witten, Phys. Lett. B155 (1985) 151.

[13] J.P. Derendinger, L.E. Ibáñez and H.P. Nilles, Nucl. Phys. B267 (1986) 365.

[14] S. Ferrara, L. Girardello and H.P. Nilles, Phys. Lett. B125 (1983) 457.

[15] H. P. Nilles, Phys. Lett. B115 (1982) 193; Nucl. Phys. B217 (1983) 366; Phys. Lett. B112 (1982) 455; Int. J. Mod. Phys. A5 (1990) 4199

[16] L.E. Ibáñez and H.P. Nilles, Phys. Lett. B169 (1986) 354.

[17] For a review see: H. P. Nilles, Phys. Reports 110 (1984) 1

[18] Y. Kawamura, H. P. Nilles, M. Olechowski and M. Yamaguchi, hep-ph9805397, JHEP 9806:008(1998)

[19] For a review see: G. Jungman, M. Kamionkowski and K. Griest, Phys. Reports 267 (1996) 195

[20] J. Conrad, hep/th-9708031, Phys. Lett. B421 (1998) 119

[21] H.P. Nilles and S. Stieberger, Nucl. Phys. B499 (1997) 3; Phys. Lett. B367 (1996) 126; S. Stieberger, Nucl. Phys. B541 (1999) 109

[22] M. Shifman and A. Vainshtein, Nucl. Phys. B277 (1986) 456.

[23] H.P. Nilles, Phys. Lett. B180 (1986) 240.

[24] Z. Lalak and S. Thomas, hep-th/9707223, Nucl. Phys. B515 (1998) 55

[25] A. Lukas, B. A. Ovrut and D. Waldram, Phys. Rev. D57(1998)7529

[26] E. A. Mirabelli and M. E. Peskin, Phys. Rev. D58(1998)065002

[27] J. Ellis, Z. Lalak, S. Pokorski and W. Pokorski, Nucl. Phys. B540(1999)149; A. Lukas, B. A. Ovrut and d. Waldram, hep-th/9901017

[28] K. Meissner, M. Olechowski and H. P. Nilles, hep-th/9905139, hep-th/9903160

[29] J. Scherk and J. H. Schwarz, Nucl. Phys. B153(1979)61

[30] I. Antoniadis and M. Quiros, Nucl. Phys. B505(1997)109 